\title{
Protocol
}

\section{Light emitting diode for the treatment of genitourinary syndrome of menopause in breast cancer survivors: study protocol for a double blinded trial}

\section{Daniela Barros ${ }^{1,2}$, Maria Clara Pavie ${ }^{2}$, Roseny Ferreira ${ }^{2}$, Cecília Alvares ${ }^{2}$, Teresa Costa ${ }^{2}$, Priscila Baqueiro $^{2}$, Ananda Machado Rego ${ }^{2}$, Emylle Anunciação ${ }^{2}$, Augusto Mariano², Júlia Lopes ${ }^{2}$, Patrícia Lordêlo ${ }^{2 *}$}

\author{
${ }^{1}$ Department of Oncology, Hospital Santa Izabel, Salvador, Brazil \\ ${ }^{2}$ Pelvic Floor Care Center (CAAP), Medicine and Human Health at the Bahiana School of Medicine and Public Health, \\ Salvador, Salvador, Brazil
}

Received: 01 May 2021

Revised: 17 May 2021

Accepted: 18 May 2021

\author{
*Correspondence: \\ Dr. Patrícia Lordêlo, \\ E-mail: pvslordelo@hotmail.com
}

Copyright: () the author(s), publisher and licensee Medip Academy. This is an open-access article distributed under the terms of the Creative Commons Attribution Non-Commercial License, which permits unrestricted non-commercial use, distribution, and reproduction in any medium, provided the original work is properly cited.

\begin{abstract}
Background: Genitourinary Syndrome of Menopause (GSM) after breast cancer are secondary to many mechanisms such long-term side effects of cancer treatment. Light-Emitting Diode (LED) is a treatment aimed at improving vaginal epithelial tissue. This study will aim to analyze the effects of $405 \mathrm{~nm}$ LED on GSM of breast cancer survivors.

Methods: This double-blind, randomized clinical trial will be conducted with breast cancer survivors aged 18-65 years, diagnosed with GSM, with at least one sign and one symptom of GSM and vaginal $\mathrm{pH} \geq 5$ or proven cytologic hypotrophy or atrophy. Pregnant women, those who received hormone replacement within the prior 6 months, with active vaginal infection, impaired comprehension, neurological disease, and progression to metastatic disease during the protocol, will be excluded. LED will be applied intravaginally with gel heated to $38^{\circ} \mathrm{C}$. Each $5 /$ weekly session will last 8 minutes, concurrent with pelvic floor muscle kinesiotherapy. The control group will be treated with the same protocol with LED device turned off. Response will be assessed with the Vaginal Maturation Index analysis, vaginal cytology, and questionnaires to evaluate urinary complains, sexual function, genital self-image and quality of life. Satisfaction will be assessed using a Visual Analog and Likert scale. Measurements will be performed pre-treatment, post-treatment week-3, and month-2 and 3 .

Conclusions: We aim to identify the benefits of LED for breast cancer survivors with GSM.

Trial registration: Clinical trials registration number NCT03833726-version 2.0, July 28, 2019.
\end{abstract}

Keywords: Breast neoplasms, GSM, LED, Photobiomodulation, Therapy

\section{INTRODUCTION}

Genitourinary Syndrome of Menopause (GSM) affects about $50 \%$ of menopausal women and up to $70 \%$ of breast cancer patients who are undergoing treatment. Symptoms are due to a decrease in hormone levels or due to blocking of circulating estrogen, inducing functional changes in the vagina such as loss of superficial cells and loss of the acidic environment.
Patients may experience chronic or permanent symptoms like pruritus, pain, burning, dysuria, erythema, vaginal dryness, dyspareunia, and fissure, even if actual menopause has not occurred. It results in the consequence of down regulation of proliferation of connective tissue, the fragmentation of elastin, and collagen hyalinization. ${ }^{1}$

This post-cancer treatment complication triggers loss of global quality of life and sexual interest and function, and 
pharmacologic interventions such as hormonal replacement are contra-indicated in breast cancer survivors. Lubricants and moisturizers are available options to help improve dryness only, but they don't provide sustained response or relieve other symptoms. ${ }^{2-4}$

Phototherapy can provide fibroblast stimulation and increase microcirculation, and recent noncontrolled trials suggest that it potentially restore epithelial integrity. ${ }^{5-8}$ Previous exploratory pilot trials have demonstrated potential benefits of intravaginal treatments. ${ }^{9}$ Research group recently published a case report that demonstrated a response to recurrent vulvovaginal candidiasis and the post-radiation treatment long-term sequelae (in press). ${ }^{10,11}$ A clinical trial for recurrent vaginal candidiasis is ongoing. We previously conducted a pilot study with noncancer post-menopause syndrome patients with promising results (in press) with three sessions with a LED device and good tolerance was observed. Now, we propose randomized trial to verify efficacy of phototherapy compared to usual kinesiotherapy treatment.

\section{Hypothesis}

We hypothesize that LED has a positive effect on cell maturation, vaginal $\mathrm{pH}$, sexual function, and quality of life in women with GSM after breast cancer treatment. ${ }^{12}$

\section{Aims of study}

Aims of study to test hypothesis that LED has a positive effect on cell maturation, vaginal $\mathrm{pH}$, sexual function, and quality of life of women with GSM after breast cancer treatment.

\section{METHODS}

\section{Study design}

Figure 1 shows spirit schedule of enrolment interventions and assessment. The LED for the treatment of GSM in breast cancer survivors (LEDCA) trial is designed as a phase II randomized, controlled, double-blind trial with 2 parallel non-stratified groups that will be performed at a specialized Pelvic Floor Care Center in Salvador - Brazil (Figure 2).

\section{Ethics approval}

This study was approved by the research ethics committee of the Bahiana School of Medicine on October 24, 2017, through CAAE 72147317.9.0000.5544 and will be in accordance with good clinical practice guidelines and Declaration of Helsinki and all participants will provide written informed consent before any protocol procedures.

This study will be funded by the Bahiana School of Medicine and Public Health, without funding from public or private entities.

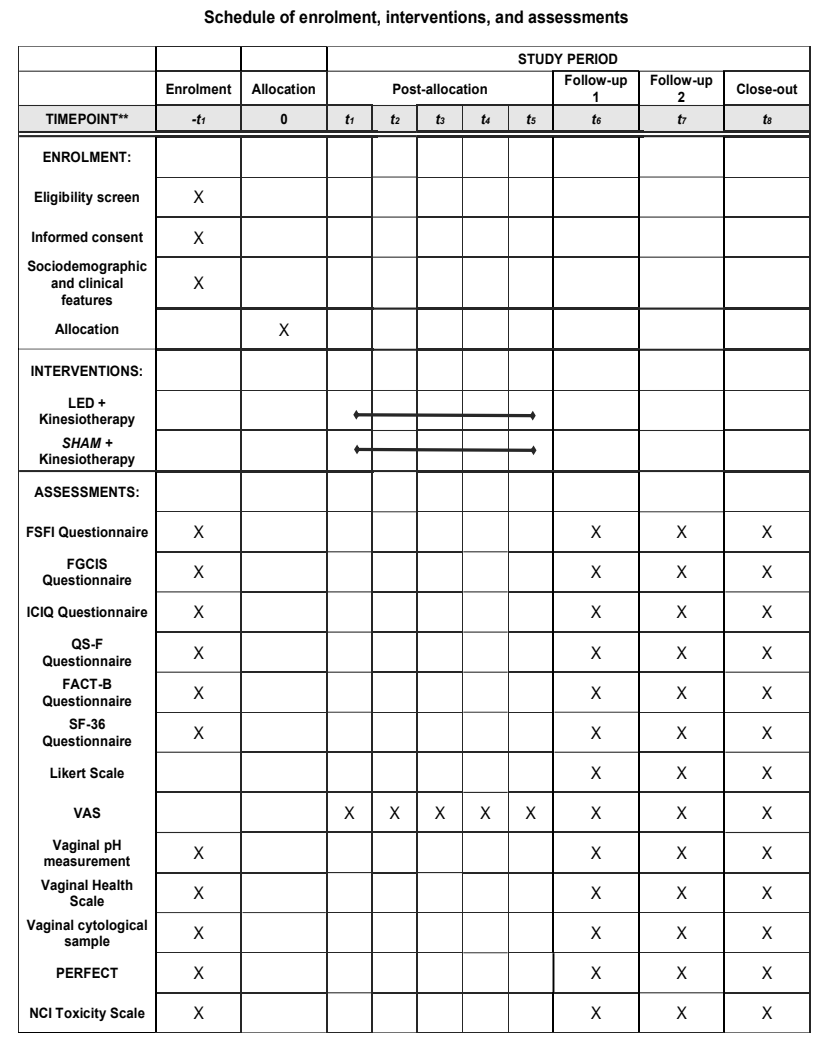

Figure 1: Spirit checklist: schedule of enrolment interventions and assessment.

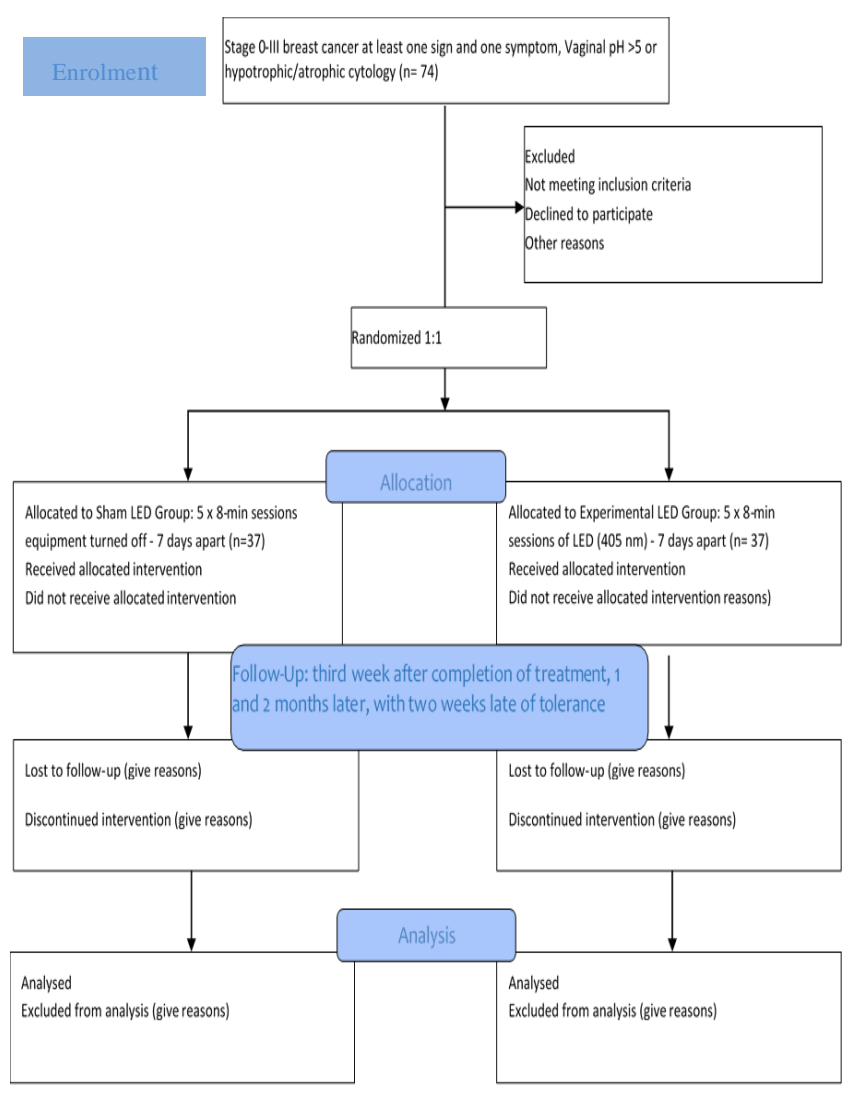

Figure 2: Study flowchart according to CONSORT. 


\section{Patients}

The study will include women aged between 18 and 65 years with pathological proven breast cancer after completion of surgical treatment, chemo and adjuvant radiation therapy classified as stage 0 -III according to the American Joint Committee on Cancer (AJCC) and International Union Against Cancer (UICC) of classification of malignant tumors-Tumor Node Metastasis (TNM) classification ( $8^{\text {th }}$ edition $) .{ }^{13}$

GSM will be diagnosed in the presence of at least one self-reported symptom and one sign identified upon gynecological examination (of external structures, introitus, and vaginal mucosa). ${ }^{3}$ If vaginal $\mathrm{pH}>5.0$, patients will be included if no exclusion criteria are met. If vaginal $\mathrm{pH}<5.0$, we will wait until vaginal cytology confirms atrophy or hypotrophy before including the patient in the study.

Patients will be excluded from the study if they have received hormonal replacement for menopause symptoms in the past 6 months, are pregnant, have a diagnosis of vaginal infection, have difficulty understanding the proposed instruments, have been diagnosed with chronic neurological degenerative diseases that preclude to stay in dorsal recumbent position, have shown progression to metastatic disease during protocol, or have received any vaginal photo application treatment in 3 months prior.

\section{Patient evaluation}

After the diagnosis of GSM is confirmed, the patient will be asked to answer a sociodemographic and clinical questionnaire, with questions about gynecological and obstetric history. To analyze sexual function, the Female Sexual Function Index (FSFI) and the Sexual Quotient-Female Version (QS-F) questionnaires will be administered. ${ }^{14,15}$ Satisfaction and confidence about self-image will be quantified using the free Female Genital Self-Image Scale (FGSIS-7). ${ }^{16}$ Urinary symptoms will be measured with the International Consultation on Incontinece Questionnaire-Short Form (ICIQ-SF). ${ }^{17}$ Aspects of quality of life will be measured with Functional Assessment of Cancer Therapy-Breast Cancer (FACT-B), medical outcomes 36-item Short-Form Health Survey (SF-36), and Visual Analog Scale Scores. ${ }^{18,19}$ FACT-B questionnaire will be used to assess treatment effect, measured by mean (or median) of questionnaire results.

Vaginal pH tape assessment, the Vaginal Maturation Index, and the Vaginal Health Scale will be assessed before patient randomization and after each reassessment. $^{20}$ The Vaginal Maturation Index will be assessed by a tertiary lab and evaluated the presence of three kinds of vaginal epithelial cells: parabasal, intermediate, and superficial. This index helps to interpret the atrophy severity, (0-49=severe, 50-64=moderate, and 65-100=discrete). The Vaginal Health Scale is a subjective scale that quantifies in numbers impression of examiner about fluids, readiness, hydration, and fragility range, scored between 0 and 25 .

Adverse events will be registered according to the Common Toxicity Criteria Adverse Events version 4.0 (CTCAE v. 4.0)-NCI toxicity scale. ${ }^{21}$ Adverse events will be classified as either possibly, probably, or definitely related to LED.

\section{Description of procedure}

Sociodemographic data will be collected including age, race, financial status, body mass index, gynecological history, urinary symptoms, sexual habits, and cancer treatments. Patients will undergo a physical exam to determine signs of vulvovaginal atrophy.

Before the first treatment, a small speculum will be inserted inside the vagina and the $\mathrm{pH}$ will be quantified using $\mathrm{pH}$ tape (MColorpHast $^{\mathrm{TM}}$, Merck, Darnstadt, Germany) held against the lateral wall for 30s. After that, a pap smear will be performed using a cytobrush and the cell samples will be placed onto a glass slide and preserved with $99 \%$ alcohol. Speculum will be removed, and pelvic floor muscle analysis will be performed using the PERFECT protocol with water-based gel. ${ }^{22}$

The intervention will comprise five sessions of treatment or sham with insertion of the device with gel heated to $38^{\circ} \mathrm{C}$ lasting 8 minutes with a 7-day interval between sessions. The LED sham sessions will follow the same pattern of treatment; however, the device will be turned off. A $405 \pm 5 \mathrm{~nm}$ blue LED (Figure 3 ) with a power of 30 $\mathrm{W}$ and a mean irradiance of $0.000773 \mathrm{~W} / \mathrm{cm}^{2}$, and consistent insertion with $38^{\circ} \mathrm{C}$ heated gel will be used. Then, patients will receive pelvic kinesiotherapy sessions as per the department protocol (2xseries of 10 sustained contractions of $5 \mathrm{~s}$ each, interchanging with $10 \mathrm{~s}$ of relaxation and $2 x$ series of maximum fast contractions, interchanging with $4 \mathrm{~s}$ of relaxation).

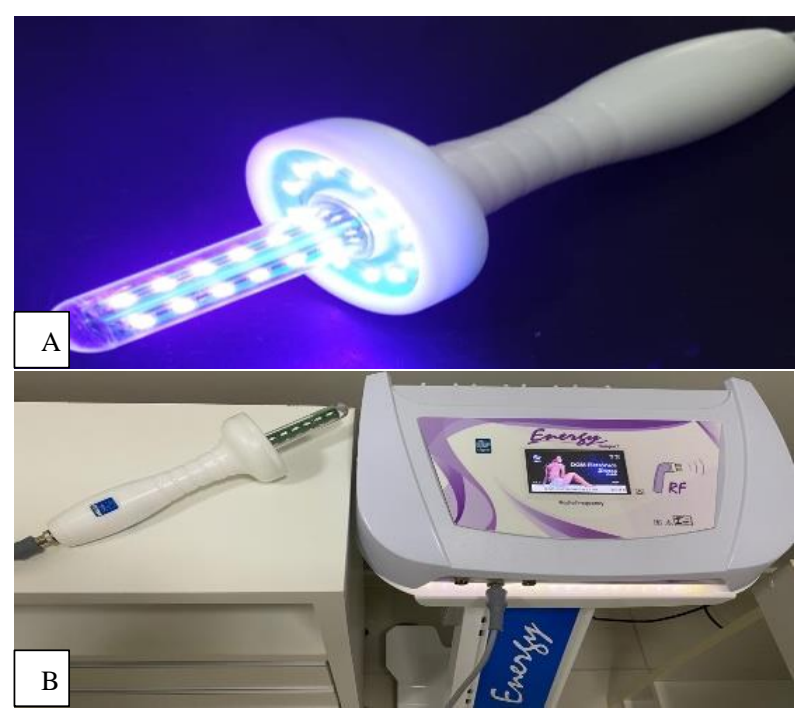

Figure 3 (A and B): Blue LED device and blue LED device on, Energy model, DGM ${ }^{\circledR}$. 
Participants will be asked to avoid applying vaginal moisturizers and lotions before treatment and asked to communicate any changes in oncological treatment. Interruptions in treatment of up to 3 weeks will be permitted. If less than 3 sessions are executed, this will be considered abandoned treatment and patients will be offered a re-start. If the patient receives three or four sessions, the patient will be considered treated. Criteria for discontinuance will be severe adverse event (grade 3 or 4) according to according to the Common Toxicity Criteria Adverse Events version 4.0 (CTCAE v. 4.0)National Cancer Institute (NCI) toxicity scale or withdrawal of consent. ${ }^{21}$ Emergency unblinding code breaks should occur in the presence of grade 3 and 4 toxicity and the patient will be sent to the gynecologist or psychologist if they feel any physical or psychological damage. Cross-over will be allowed if we can identify the benefits.

Randomization will be performed by a staff member who will not participate any further in the research, and sequences will be created using Microsoft excel for windows 2007 (Microsoft Corp., Redmond, WA, USA) and will be unknown to the investigator. Due to the nature of the intervention, the staff responsible for executing the intravaginal treatment can not be blinded to allocation, but the patient, data collectors, and staff responsible for data analysis will be blinded to treatment allocation.

The patients will be randomly divided $1: 1$ to either the LED group or LED Sham group. Both patients and kinesiotherapy physiotherapists will not be aware of treatment allocation. Only the physiotherapist who executes the LED procedure will have access to allocation information.

\section{Evaluation of therapeutic response}

The evaluations will be scheduled for the third week after completion of treatment, 1 month later ( 7 weeks after treatment), and 2 months later, (15 weeks after treatment), with two weeks late of tolerance.

The influence that missing data may have on the results will be assessed via a sensitivity analysis of augmented data sets. Dropouts (essentially, patients who withdraw consent for continued follow-up) will be included in the analysis using modern imputation methods for missing data.

\section{Interim analysis}

An interim analysis will be performed on the primary endpoint when $50 \%$ of patients have been randomized and have completed 3 months of follow-up. The interim analysis will be performed by an independent statistician blinded to treatment allocation and published as partial results reporting.
To assess domains of sexual functioning (e.g., desire, sexual arousal, lubrication, orgasm, satisfaction, pain, or discomfort) and estimate female sexual dysfunction, we will use the FSFI Full scale score, validated for the Brazilian Portuguese population. The FSFI has a range of scores between 2 and 36 and is a brief, multidimensional questionnaire used to assess key dimensions of sexual function in women.

A free translation to Brazilian Portuguese of Sexual Quotient-Female Version (QS-F), which is a ten-question life domain with a total score ranging from 0 to 100 , will be used to screen for sexual dysfunction. A score between 82 and 100 indicates good to excellent, 62 and 80 indicates fair to good, 42 and 60 indicates unfavorable to fair, 22 and 40 indicates bad to unfavorable, and 0 to 20 indicates null to bad.

Satisfaction and confidence in self-image will be quantified using a free translation to Brazilian Portuguese of the 7-Question Female Genital Self-Image Scale (FGSIS-7). Scores range from 7-28 and higher scores indicate better self-image.

Urinary symptoms will be measured with the ICIQ-SF, validated for the Brazilian Portuguese population.

Aspects of quality of life will be measured using FACTB, SF-36, and VAS scores. The FACT-B questionnaire validated for the Brazilian Portuguese population rates quality of life in terms of physical (PWB), social/family (SWB), emotional (EWB), functional wellbeing (FWB), breast cancer subscale (BCS), and arm subscale (ARM) aspects. The total score range is 0 to 148 and it is calculated by adding the subscales (PWB with SWB, EWB, FWB and BCS) and higher scores indicate better quality of life.

The SF-36 validated for the Brazilian Portuguese population will also be used and compared with FACT-B results. The SF-36 has a range of scores between 0 and 100 , it analyzes self-care ability, pain, vitality, and mental status including mood and wellbeing, and lower values indicate poorer quality of life.

At each study visit, patients will be questioned about adverse events they have experienced since the last study visit, and patients will be analyzed in accordance with the appearance of symptoms during treatment. Selfadministered questionnaires will be administered before each session using a VAS with a range 0 to $10(0=$ no symptoms, $10=$ severe symptoms) to determinate tolerance, toxicity, dryness, dyspareunia, pruritus, laxity, burning, and introitus pain.

A Likert scale will be used at reassessment to measure the individual's satisfaction, scaled from 1 to 5 (1=not happy at all with the treatment, 5=very happy with the treatment). 
Vaginal $\mathrm{pH}$ tape measurement will be performed before randomization and physical exam and after each reassessment.

The maturation vaginal index will be assessed by a tertiary lab and will evaluate the relationship between the three kinds of vaginal epithelial cells: parabasal, intermediate, and superficial. This index helps to interpret the atrophy severity ( 0 to $49=$ severe, 50 to $64=$ moderate, and 65 to $100=$ discrete).

Vaginal health is a subjective scale that quantifies in numbers the impression of the examiner in terms of fluids, readiness, hydration, and fragility range 0 to 25 , and will be performed before randomization, physical exam, and after each reassessment.

Patients will be informed about potential risks and harms including burning sensation, itching, and temporary increase of vaginal discomfort.

Adverse events will be registered and graduated according to the Common Toxicity Criteria Adverse Events version 4.0 (CTCAE v. 4.0)-NCI toxicity scale. Adverse events will be classified as either possibly, probably, or definitely related to LED.

\section{Data management}

Patient files will be registered in numerical order and stored in a secure and accessible place and manner. Paper and electronic patient files will be maintained in storage for a period of 5 years after completion of the study and the authors will be responsible for access control and draft writing.

\section{Statistics}

For the elaboration of the database, and descriptive and analytical analysis, the Software Statistical Package for Social Sciences (SPSS) version 14.0 for Windows (IBM Corp., Armonk, NY, USA) will be used. The normality of the variables will be verified through descriptive statistics and the Shapiro-Wilk test. Categorical variables will be expressed as absolute values and percentages (n [\%]), continuous variables with normal distribution will be expressed as mean and standard deviation (mean $\pm \mathrm{SD}$ ), and with asymmetric distribution, as median and interquartile range. Unpaired inferences and paired inferences will be analyzed using a Chi-Square test $\left(\mathrm{x}^{2}\right)$ or a Fisher's exact test, respectively. For paired intragroup analyses, a paired student's t test or Wilcoxon test will be used. For unpaired intergroup analyses, the Student's t test or Mann-Whitney $U$ test will be used depending on normality. The level of significance will be $5 \%$.

This protocol publication intends to grating public access to full protocol, and once all analysis completed, data will be sent to appropriate publication.

\section{Sample size}

We estimated sample size using a Winpepi calculator v. 11.65. Results showed that a sample of 74 patients, 37 in each group, inflated to compensate for $10 \%$ non-inclusion rate or loss (based on the study by Yaralizadeh et al and a SD of 15.3 in the treatment group and SD of 8 in the control group would detect a 10-point difference in the vaginal cell maturation index with an alpha error of 0.05 and power of $80 \% .^{23}$

For co-primary analysis of sexual quality of life, we estimated a sample size of 58 patients for a detectable difference of 5 points on the FSFI with an SD of 7.7 and 5.6 and an alpha error of 0.05 and power of $80 \%$.

\section{DISCUSSION}

Because it has a clinical trial methodology, the execution may provide proof of the safety and efficacy of a conservative and non-medicated therapeutic protocol, in addition to the use of a product with a patent by a physical therapist researcher (patent deposit number BR 102017026980 9). It is noteworthy that the technique, in addition to the device, was developed by the group and will be of great value to physiotherapists, as it will be another resource for performance in the area of women's health, representing an important impact on the marketing aspect for professionals graduated in physiotherapy.

Since the hormonal mechanisms of breast cancer carcinogenesis have been discovered, endocrine therapy has been used as a strategy to increase cancer control and promote a lower risk of recurrence; however, hormone therapy evokes menopausal symptoms as common late toxicity prevents endogen estrogens from functioning properly and promotes down regulation of hormone levels. The limitations of the available treatments for symptom relief have encouraged researchers to seek strategies to treat GMS and will not increase the systemic risk of recurrence. ${ }^{3,4,6}$

Substantial evidence demonstrates that local treatment with fractional LASER devices such as CO2 or Er:YAG on the skin and intravaginally propels recovery of epithelium thickness and tissue vascularization, and improves quality of life and sexual symptoms. However, it has not yet been established how many sessions are needed and the optimal duration of each session for safe and effective treatment. ${ }^{8}$ Despite the benefits, these treatments are expensive and bothersome, so treatment based on an alternative light-based device may be a more comfortable and less expensive method for achieving similar result.

A previous multicenter study using blue LED (415 nm), red LED $(660 \mathrm{~nm})$, and infrared LED $(850 \mathrm{~nm})$ showed promising results on epithelial maturation and also on FSFI scores; however, patients were only followed up for a short time and there was no control group. ${ }^{9}$ 
We hypothesized that the potential to evoke blood flow could promote local neovascularization and neocollagenesis; however, the relationship between the severity of complaints and the severity of decidua changes is not known, so we plan to compare the different parameters simultaneously, such as vaginal $\mathrm{pH}$, the Vaginal Health Index, the Vaginal Maturation Indedx, VAS, and validated questionnaires in order to investigate the predominant complaints, such dryness perception, pruritus, dyspareunia, and urinary symptoms.

\section{CONCLUSION}

In conclusion, the expected outcome of this prospective placebo controlled clinical trial is to demonstrate the subjective and objective benefits of this feasible local treatment that can be implemented in clinical practice as a safe alternative treatment method for oncologic patients with GSM.

\section{ACKNOWLEDGEMENTS}

All authors would like to thank the members of the Pelvic Floor Care Center (CAAP) and the Bahiana School of Medicine and Public Health. The government institutions CNPQ and FAPESB for promoting research.

Funding: No funding sources Conflict of interest: None declared

Ethical approval: The study was approved by the Institutional Ethics Committee of Bahiana School of Medicine

\section{REFERENCES}

1. Portman DJ, Gass MLS. Genitourinary Syndrome of Menopause: New Terminology for Vulvovaginal Atrophy from the International Society for the Study of Women's Sexual Health and The North American Menopause Society. J Sex Med. 2014;11:2865-72.

2. Kyvernitakis I, Ziller V, Hars O, Bauer M, Kalder M, Hadji P. Prevalence of menopausal symptoms and their influence on adherence in women with breast cancer. Climacteric. 2014;17(3):252- 9.

3. Moreno AC, Sikka SK, Thacker HL. Genitourinary syndrome of menopause in breast cancer survivors: Treatments are available. Cleve Clin J Med. 2018;85(10):760-6.

4. Lester J, Pahouja G, Andersen B, Lustberg M. Atrophic vaginitis in breast cancer survivors: a difficult survivorship issue. J Pers Med. 2015;5(2):50-66.

5. Lanzafame RJ, De la Torre S, Leibaschoff GH. The Rationale for Photobiomodulation Therapy of Vaginal Tissue for Treatment of Genitourinary Syndrome of Menopause: An Analysis of Its Mechanism of Action, and Current Clinical Outcomes. Photobiomodulation, Photomedicine Laser Surg. 2019;37(7):395-407.
6. Sussman TA, Kruse ML, Thacker HL, Abraham J. Managing Genitourinary Syndrome of Menopause in Breast Cancer Survivors Receiving Endocrine Therapy. J Oncol Pract 2019;15(7):363-70.

7. Opel DR, Hagstrom E, Pace AK, Sisto K, Hirano-Ali SA, Desai $\mathrm{S}$ et al. Light-emitting diodes: a brief review and clinical experience. J Clin Aesthet Dermatol. 2015;8(6):36-44.

8. Arroyo C. Fractional $\mathrm{CO} 2$ laser treatment for vulvovaginal atrophy symptoms and vaginal rejuvenation in perimenopausal women. Int $\mathbf{J}$ Women's Health. 2017;9:591-5.

9. García PN, Elias JA, Parada JG, Luciañez DZ. Management of Vaginal Atrophy with Intravaginal Light-Emitting Diodes (LEDs). Pint H Int J Obst Gynaecolo Res. 2018;5(2):632-41.

10. Pavie MC, Robatto M, Bastos M, Tozetto S, VilasBoas A, Vitale SG, Lordelo P. Blue light-emitting diode in healthy vaginal mucosa-a new therapeutic possibility. Lasers Med Sci. 2019;34(5):921-7.

11. Robatto M, Pavie MC, Tozetto S, Brito MB, Lordêlo $\mathrm{P}$. Blue light emitting diode in treatment of recurring vulvovaginal candidiasis: a case report. Brazilian J Med Human Health. 2017;5(4):162-8.

12. Nilsson K, Risbergb B, Heimerc G. The Vaginal Epithelium in the Postmenopause: Cytology, Histology and $\mathrm{Ph}$ as Methods of Assessment. Maturitas. 1995;5122(94):51-6.

13. American Joint Committee on Cancer. AJCC Cancer Staging Manual- $8^{\text {th }}$. Chicago-Il. Springer. 2017;983.

14. Hentschel H, Alberton DL, Capp E, Goldim JR, Passos EP. Validação Do Female Sexual Function Index (FSFI) Para Uso Em Língua Portuguesa Revista HCPA. 2007;27(1):10-4.

15. Helena C, Abdo N. Quociente sexual feminino: um questionário brasileiro para avaliar a atividade sexual da mulher. Medibina Sex. 2009;14(2):89-91.

16. Herbenick D, Reece M. Development and validation of the female genital self-image scale. J Sex Med. 2010;7(5):1822-30.

17. Tamanini JTN, Dambros M, D’Ancona CAL, Palma PCR, Rodrigues Netto NJr. Validação para o português do "International Consultation on Incontinence Questionnaire-Short Form" (ICIQ-SF). Rev Saúde Pública. 2004;38(3):438-44.

18. Michels FAZ, Latorre MRDO, Maciel MS. Validação e reprodutibilidade do questionário $\mathrm{FACTB}+4$ de qualidade de vida específico para câncer de mama e comparação dos questionários IBCSG, EORTCBR23 e FACT-B+4. Cad Saúde Colet. 2012;20(3):321-8.

19. Ciconelli RM, Ferraz MB, Santos W, Meinão I, Quaresma MR. Tradução e validação do SF36. Rev Bras Reumatol. 1999;39(3):143-50.

20. Bachmann G. Urogenital ageing: an old problem newly recognized. Maturitas. 1995;22:S1-5.

21. U.S. Department of Health and Human Services. Common Terminology Criteria for Adverse Events (CTCAE) 2017;5.0.

22. Laycock J, Jerwood D. Pelvic Floor Muscle 
Assessment: The PERFECT Scheme. Physiotherapy. 2001;87(12):631-42.

23. Yaralizadeh M, Abedi P, Najar S, Namjoyan F, Saki A. Effect of Foeniculum vulgare (fennel) vaginal cream on vaginal atrophy in postmenopausal women: A double-blind randomized placebo-controlled trial. Maturitas. 2016;84:75-80.
Cite this article as: Barros D, Pavie MC, Ferreira R, Alvares C, Costa T, Baqueiro P, et al. Light emitting diode for the treatment of genitourinary syndrome of menopause in breast cancer survivors: study protocol for a double blinded trial. Int J Clin Trials 2021;8(3):211-7. 